\title{
Wind Power Forecast Uncertainty in Daily Operation of Wind Park Combined with Storage
}

\author{
Hrvoje Keko, Mauro Augusto da Rosa, Jean Sumaili, Vladimiro Miranda \\ INESC Porto \\ Campus da FEUP, Rua Dr. Roberto Frias 378, 4200-465 Porto, Portugal \\ hrvoje.keko@inescporto.pt, mauro.rosa@inescporto.pt \\ jean.sumaili@inescporto.pt, vladimiro.miranda@inescporto.pt
}

\begin{abstract}
The inevitable wind power forecast errors result in differences between forecasted and observed wind power. To mitigate their economic impact, combining the wind power with pumped hydro energy storage may be used. In order to deliver a joint operational strategy for a wind power plant combined with storage, one requires reliable wind power forecasts. The forecasts commonly only consist of a single-value forecast (point forecast) for each look-ahead time. However, additional representative information concerning the forecast uncertainty may be required. In this paper, the influence of uncertainty representation daily operation for a wind power plant combined with pumped hydro storage is discussed. The paper illustrates how uncertainty representations such as Gaussian uncorrelated errors may also not be satisfactory. Based on a case study with a wind power plant combined with pumped hydro storage, organized for illustrative purposes, the paper demonstrates the need to have uncertainty representation including cross-period dependencies, in order to define a correct operation policy.
\end{abstract}

\section{INTRODUCTION}

The stochastic nature of wind power production increases variability and volatility in electric power systems, which poses a challenge for power system operation and planning. The research efforts are constantly focused on increasing the forecast accuracy; however imperfect prior knowledge makes perfect forecasting practically impossible. A recent extensive overview of the state of the art in wind power forecasting can be found in [1].

The forecasting errors result in differences between observed wind power values and the forecasted ones. If wind power operators are participating in the electricity market, these forecast errors directly affect the wind park operator profits. When guaranteed feed-in tariffs for wind power are in place (e.g. Portugal, where off-peak hours are less remunerated than the peak hours, using a hourly modulation coefficient [2]), the forecast discrepancies may affect the owner profit expectations. The errors may also affect market prices and security of operation.

The actual influence of forecast errors is subject to local market rules - in some occasions biased imperfect forecasts may be favored ahead of the "perfect" ones. A discussion on such desired properties of forecasts can be found in [3].

In order to mitigate the impact of forecast imperfections, a technique of combining the wind power with energy storage may be used. Depending on the market, multiple roles of the energy storage may be foreseen. Energy may be stored during low price periods and released when prices are higher, thus the storages would help in taking advantage of price variations. On the other hand, storage may be used in order to increase the share of energy produced by wind, so that energy is stored when wind is abundant and later released when required [4].

To define a joint wind-hydro storage operational strategy, one requires adequate wind power forecasts. These commonly consist only of predictions characterized by a single-value forecast (point forecast) for each look-ahead time. The information thus provided by forecast is a conditional expectation for each look-ahead time. However, there is also uncertainty associated which should be taken into account in the decision making process. Several models of uncertainty representation exist, recently reviewed in [1]. Probabilistic forecasts [5] can be used, presented either by moments of probability density function, by using a set of quantiles (a common case) [6], by defining a probability mass function, or by stating a full probability density function as the most general case. Another option is to use risk indices [7] as a measure of forecast uncertainty.

One of the problems with the models referred to above is that these are developed for each look-ahead time independently. In effect, these models do not to provide the information on the evolution of forecast uncertainty throughout the period in analysis. The time dependency of forecast errors is not taken into account, and ignoring this dependency may cause overestimation or underestimation of the estimated risks. In [8], a non-parametric method for generation of statistical scenarios for short-term wind power production is presented, respecting the interdependence structure. This is especially relevant when the analyses include energy storage [9]. Namely, including storage transforms the system from one with "no memory" to a system with memory, since the storage device energy content represents an integral over the previous operational period. The study in [4] confirms that adding pumped storage to a wind power plant can have a favorable effect on the combined wind-hydro profits. Furthermore, for the operational purposes, when the system includes storage, it is argued that one should use an uncertainty modeling method capable of including temporal (and even spatial) interdependence structure of uncertainty, and that neglecting it may lead to strong under- or overestimation of the uncertainty. This is discussed, for example, in [9].

This paper delivers a comparison of two uncertainty modeling approaches in the context of the optimization of a 
daily operation for a wind power plant combined with smallscale pumped hydro storage. In the following sections, firstly the formulation of optimization model of a wind-hydro power plant is presented, and then the two uncertainty models being compared are presented. Finally, test case study results along with conclusions are provided.

\section{Optimization Model of a Wind-Hydro Power Plant}

The pumped hydro storage functions as an "energy hedging" mechanism and its function is twofold: storing energy during low price periods to release it during high price periods, or complementing the wind power gaps in order to keep the total production within desired limits.

In this paper the optimization model follows the one presented in [4]: it is an hourly-discretized optimization algorithm identifying the optimum daily operational strategy to be followed by wind turbines and pumped hydro equipment. The objective function can be tuned either to minimize the fluctuations or maximize the total operational profit. In this paper, the profit maximization is considered.

The problem is modeled as a linear optimization problem. The function to be maximized is the total 24-hour (daily) operational profit of the wind-hydro (W-H) power plant. For each of scenarios, i.e. for each time series of 24 forecasted wind power productions the following problem is solved:

Maximize

$$
V A L=\sum_{i=1}^{n}\left(c_{i} P_{i}-c_{p} P p_{i}\right)+n c_{\alpha} \alpha
$$

subject to: $(i=1 . . n)$

$$
\begin{gathered}
P_{i}=P w_{i}+P h_{i} \\
P v_{i}=P w_{i}+P p_{i}+P_{D L i} \\
E_{i+1}=E_{i}+t\left(\eta_{p} P p_{i}-\frac{P h_{i}}{\eta_{h}}\right) \\
E_{1}=E_{\text {begin }} \\
E_{n+1}=E_{\text {end }} \\
\alpha P_{i}^{L} \leq P_{i} \leq P_{i}^{U} \\
P g^{L} \leq\left(P w_{i}+P p_{i}\right) \leq P g^{U} \\
P h^{L} \leq P h_{i} \leq \min \left(P h^{U}, \frac{\eta_{h} E_{i}}{t}\right) \\
P p^{L} \leq P p_{i} \leq P p^{U} \\
0 \leq E_{i} \leq E^{U} \\
0 \leq \alpha \leq 1.0 \\
P_{D L i} \geq 0
\end{gathered}
$$

The hourly variables are: $P$-active powers delivered to the network; $P W$ - active powers delivered by the wind turbine; $P h-$ active powers produced by hydro generator, $P p$-active powers consumed by pumping equipment, $P_{D L}-$ curtailed (unused) part of the wind power curve i.e. a part of the wind power not used to generate electricity even though it is available; $E$ - energy storage levels. The variable $\alpha$ represents a decrease factor in lower output limit, and $\eta_{p}$ and $\eta_{h}$ represent the efficiencies of pumping and hydro turbine equipment, respectively.

The superscript $L$ indicates the lower and superscript $U$ the upper limit, for $P g$ (capacity limits of the wind park), $P h$ (hydro generator limits), $P p$ (pumping equipment limits) and $E$ (energy storage limits).

The problem is further constrained by the minimum and maximum total output power $P^{L}$ and $P^{U}$ and these may be related to market or network requirements.

The objective function has two parts: the first tries to maximize the total operational profit and the second tries to keep the minimum desired hourly production that may represent the contractual obligation or bid in the market. The vector $c$ defines the hourly active power prices, $c_{p}$ the pumping operation internal cost, and $c_{\alpha}$ is the penalization cost for failing to deliver the minimum output limit. This factor may reflect the power that the operator needs to buy at the real-time market to compensate for the unavailable power. If $c_{\alpha}$ is set much larger than $c_{p}$ and $c$, then the optimization algorithm will try to increase the lower output limit of the combined facility: effectively, the minimum output limit is set as soft constraint. If the scheduled lower bound limit cannot be met, this constraint is relaxed, compensating with the costs of energy bought in the market. In the presented case study, no lower limit was imposed, i.e. $P^{L}=0$. The operation is optimized on a daily basis and in this model it is considered that the desired reservoir levels at the beginning of the day $E_{\text {begin }}$ and at the end of the day $E_{\text {end }}$ are both known in advance. The final desired level $E_{\text {end }}$ may be determined by extending the simulation horizon to a longer period, e.g. two days, and then considering only the first day operationally.

In the formulation of the above problem, a vector of forecasted available wind power $P v$ is required. For a given $P v$, the results of the optimization problem are the hourly active powers to be generated by wind and hydro generators. The two procedures of obtaining $P v$ scenarios are described in the following section. The above model is implemented using ILOG CPLEX solver by means of its Python interface.

\section{STOCHASTIC MODEL OF THE WIND POWER}

In the problem formulation, $P v$ is a vector of forecasted wind powers for the following day. Let $\hat{p}_{t+k \mid t}$ denote a point forecast for the time $t+k$ delivered at time $t$. In this case $P v$ contains $\hat{p}_{t+k \mid t}$ for $k=0.24$ so that a time series of 24 hourly values of forecasted wind are observed as a single scenario.

Given that the accuracy of point forecasts is highly variable, various methods have been devised to estimate the uncertainty of the forecasts. Two stochastic models for the wind power are tested in this work and both models do not depend on the actual forecasting method used to deliver the point forecasts.

The first model is similar to the one employed in the simulations in [4]: the scenarios of wind power are generated based on the assumption of Gaussian distribution: the point forecast is used as the mean and the standard deviation is estimated from historic forecast performance. The standard deviation is considered to vary from hour to hour, i.e. the accuracy of $\hat{p}_{t+k \mid t}$ is considered to depend on the look-ahead horizon $k$. In other words the point forecast is "dressed" with 
a Gaussian distribution; however the samples are sampled independently for each hour.

The conversion of wind speed to wind power through wind power curve is a highly nonlinear process. This contributes to the forecast error distribution shapes that can, at best, be assumed to only weakly follow known parametric distributions. In particular, recent developments in short-term wind power forecasting indicate that the error distribution is cannot be assumed Gaussian. In [10], the authors present a non-parametric method that does not require assumptions of Gaussianity for the error distribution.

Sampling the hours separately does not inform on the forecast uncertainty development. The second model tested in this paper sees the realized measured power value at time $t+k p_{t+k}$ as a realization of the random variable $P_{t+k}$, whose probability density function is $f_{t+k}$. A probabilistic forecast delivered at time $t$ for time $t+k$ provides an estimate of the probability density function $\hat{f}_{t+k \mid t}$.

In [8], the authors present a method of generating statistical scenarios that respects the marginal density function of the forecast, taking into account the interdependence structure of prediction errors. No assumption is made on the shape of predictive distribution $\hat{f}_{t+k \mid t}$. Starting with nonparametric probabilistic forecasts (for each look-ahead time), such forecasts are suitably transformed to a Gaussian domain. The interdependence structure of the forecast errors is embedded in a recursively estimated covariance matrix (estimated over past forecast performances). This estimated covariance matrix captures the information on the interdependence of individual hourly realizations. It is then used to generate a scenario (i.e. a sequence of power values) starting from a seed, obtained by sampling a multivariate Gaussian distribution and then applying an inverse transformation to the one that converted the errors to Gaussian. It should be noted that the covariance matrix is the simplest assumption and more complex dependence structures may be employed (e.g. copulae [11]). In this paper, the method will be referred to as time-dependent scenario sampling method.

\section{CASE STUDY DATA}

\section{A. Technical Data}

Table I gives the technical data necessary to formulate the optimization problem described in Section II.

TABLE I

Wind-Hydro Power Plant TeChNicAl Characteristics

\begin{tabular}{c|c|c|c|c}
\hline $\begin{array}{c}P g^{U} \\
{[\mathrm{MW}]}\end{array}$ & $\begin{array}{c}P h^{U} \\
{[\mathrm{MW}]}\end{array}$ & $\begin{array}{c}P p^{U} \\
{[\mathrm{MW}]}\end{array}$ & $\begin{array}{c}c_{\alpha} \\
{[€ / \mathrm{MWh}]}\end{array}$ & $\begin{array}{c}c_{p} \\
{[€ / \mathrm{MWh}]}\end{array}$ \\
\hline 12 & 3 & 3 & 500 & 1.5 \\
\hline \multicolumn{5}{|l}{} \\
\hline$E^{U}$ & $\begin{array}{c}E_{\text {begin }} \\
{[\mathrm{MWh}]}\end{array}$ & $\begin{array}{c}E_{\text {end }} \\
{[\mathrm{MWh}]}\end{array}$ & $\eta_{h} \cdot \eta_{p}$ & $\begin{array}{c}c_{p} \\
{[€ / \mathrm{MWh}]}\end{array}$ \\
\hline 24 & 0 & 0 & 0.75 & 1.5 \\
\hline
\end{tabular}

The global hydraulic circuit efficiency is set to 0.75 , which corresponds to a typical value. The pumping operation $\operatorname{cost} c_{p}$ only represents the pump costs while the efficiency is considered separately. The desired hydro storage level is set to
0 both at the beginning and at the end of the simulated period. This means energy hedging can only happen within the studied day.

\section{B. Wind Power Forecast Dataset}

Wind power forecast data used in this study are freely available day-ahead wind power point forecasts and actual generation from US National Renewable Energy Laboratory's Eastern Wind Integration and Transmission Study (EWITS) [12]. These forecast data were produced by combining a mesoscale weather model with a composite power curve for a number of potential sites for wind power farms in the United States. The complete information on the methodology can be found in [13]. The observed period corresponds to one year of data (2006). The first six months, January to June 2006, are used for analysis - i.e. estimation of standard deviations in the Gaussian scenario generation method (Fig. 1) and initial covariance matrix estimation in the scenario generation method from [8] (Fig. 2).

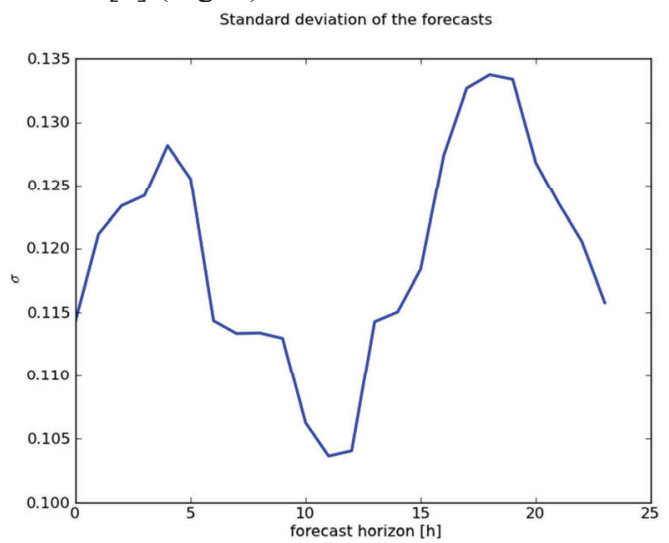

Fig. 1. Observed standard deviations used in Gaussian dressing-based scenario sampling

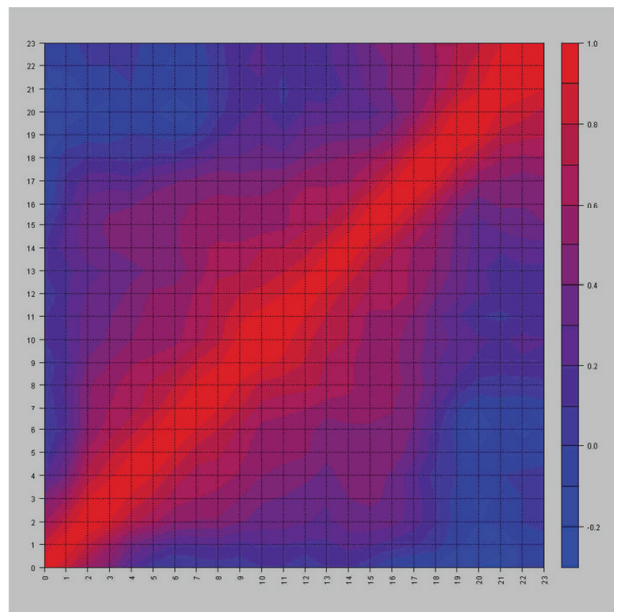

Fig. 2. An (initial) color gradient estimate of $24 \times 24$ hour covariance matrix based on forecast performance from January to July 2006, used in timedependent scenario sampling. Note how the highest correlation is achieved between adjacent hours, and in some hours the dependence structure is more complex than in others.

In both cases it is assumed that the future forecast performance will resemble the recent one. Figures 3 and 4 
illustrate the scenario sampling process, by the hourly independent method and the time-dependent scenario sampling. Besides the actual scenarios, in both figures an envelope of minimum and maximum sampled value for each hour is indicated, as well. In both figures, the scenarios are sampled for the same day, i.e. the point forecast and realized wind power time series are the same.

Per-hour independent scenario sampling

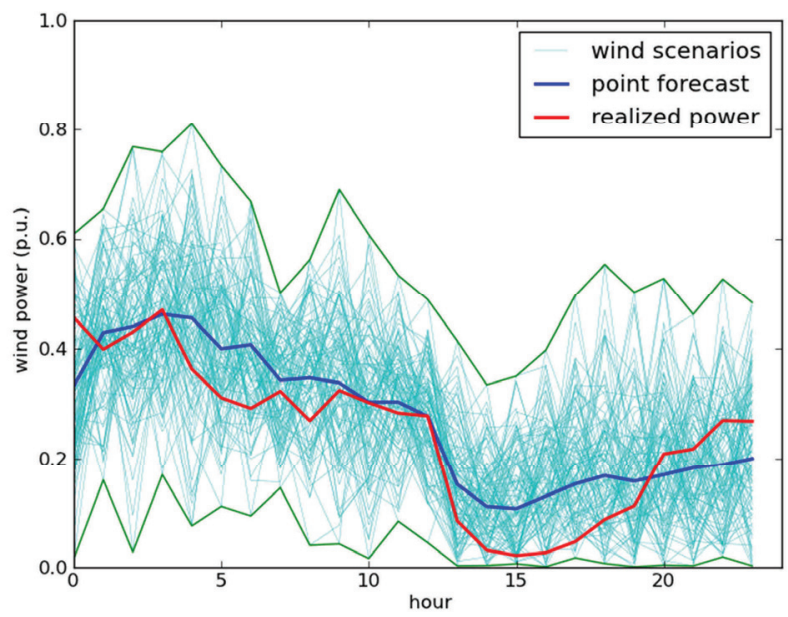

Fig. 3. A set of 100 wind power scenarios sampled using per-hour independent scenario sampling method

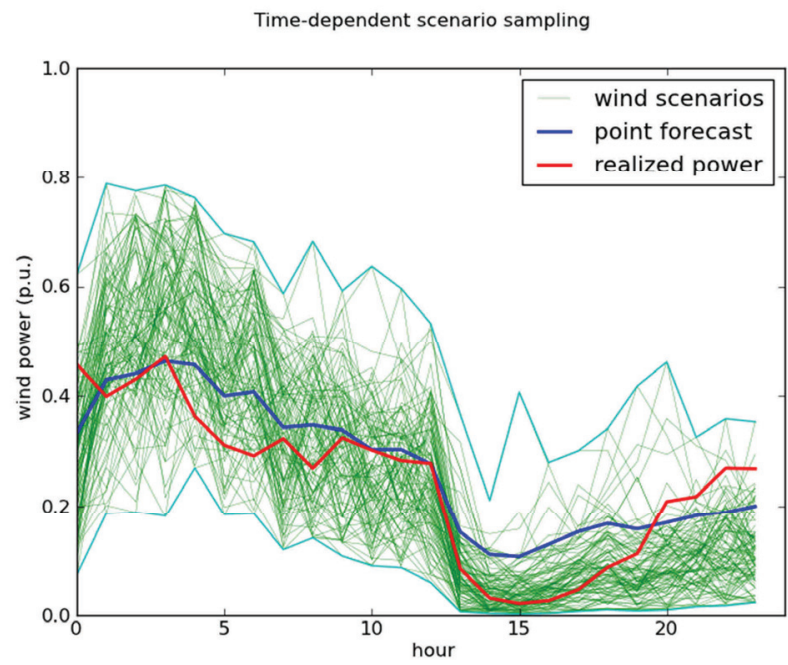

Fig. 4. A set of 100 wind power scenarios sampled using the time-dependent scenario sampling method

The visual inspection of the graphs indicates the distribution of scenarios is different and the difference is even more obvious if a histogram of the sampled scenarios is inspected. An illustration is depicted in figures 5 and 6 . The histograms present the distribution of sampled wind power for the same day depicted in figures 3 and 4, for 18 hours ahead.

Note, however, that without the actual simulations, the only prior conclusion that can be drawn is that the results of the hourly independent and time-dependent scenario sampling will be different. The actual representativeness of scenarios needs to be confirmed in a simulation.

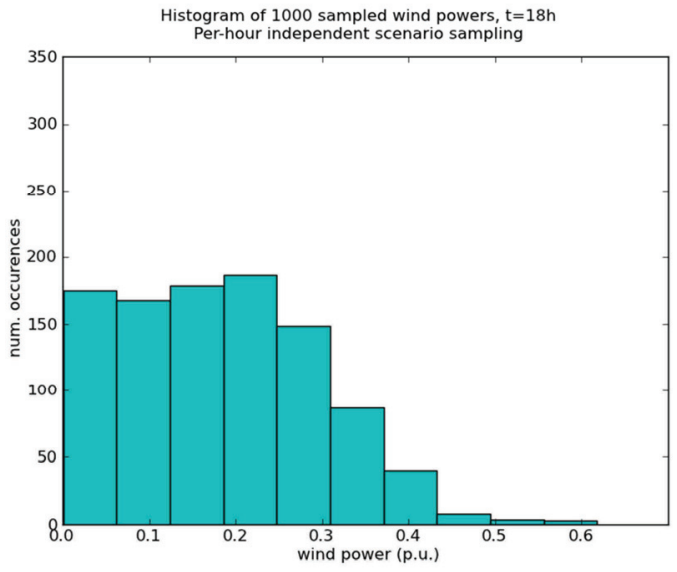

Fig. 5. A histogram illustrating the distribution of 1000 wind power scenarios sampled using the per-hour independent scenario sampling

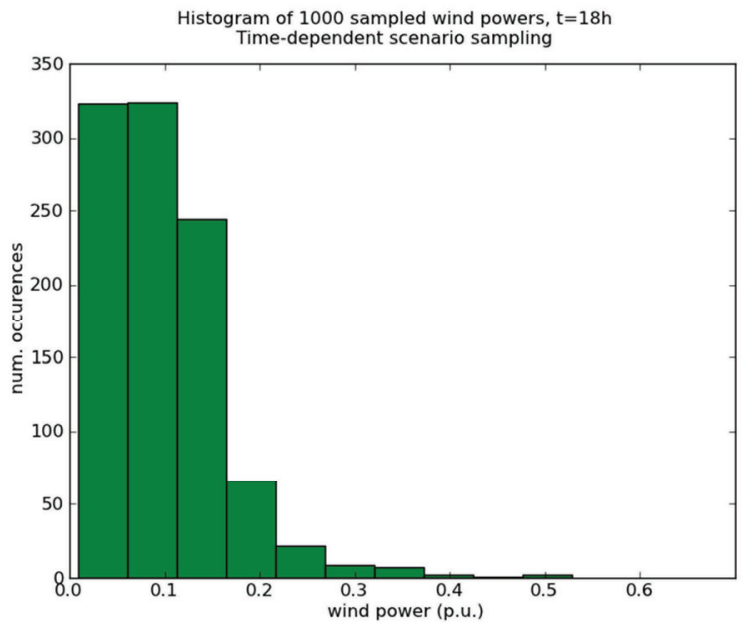

Fig. 6. A histogram illustrating the distribution of 1000 wind power scenarios sampled using time-dependent scenario sampling

\section{Pricing / Remuneration Formulation}

Since the hydro storage is only able to store energy coming from wind power, all the $\mathrm{W}-\mathrm{H}$ production is considered to be remunerated as wind production. In Portugal, this would be favorable for the $\mathrm{W}-\mathrm{H}$ plant since wind is better remunerated than hydro power. In the illustrative case study presented in this paper, the prices during the peak hours period are doubled, so that $c_{i}=50 € / \mathrm{MWh}$ for $0 \leq i<8$ and $22 \leq i<24$, and 100 $€ / \mathrm{MWh}$ for $8 \leq c_{i}<22$. This way the optimization algorithm may take advantage of higher price in peak periods.

\section{Simulation Results}

In the first simulation, 1000 scenarios are sampled for a single day using both methods. Each of the sampled scenarios is included in the optimization as the vector $P v$ of the forecasted wind powers $\hat{p}_{t+k \mid t}$ for $k=1 . .24$. In other words for each of the 1000 scenarios an optimal operational strategy is determined, as if this were the real wind power. This means the optimization model converts the random vector $P v$ into a 
new random variable $V A L$ represented by a discrete sample of profit values. For both methods of scenario sampling, Table II summarizes the obtained results, if no storage capability is considered.

TABLE II

A Single Day 1000 Scenario Simulation Results

Wind Power ONLY TOtal DaIly Profits

\begin{tabular}{c|c|c|c}
\hline $\begin{array}{c}\text { Scenario } \\
\text { gen. } \\
\text { method }\end{array}$ & $\begin{array}{c}\text { Min. } \\
\text { profit } \\
{[€]}\end{array}$ & $\begin{array}{c}\text { Avg. } \\
\text { profit } \\
{[€]}\end{array}$ & $\begin{array}{c}\text { Max. } \\
\text { profit } \\
{[€]}\end{array}$ \\
\hline $\begin{array}{c}\text { Hourly } \\
\text { indep. } \\
\text { sampling }\end{array}$ & 4598 & 6301 & 8269 \\
\hline $\begin{array}{c}\text { Time- } \\
\text { dep. } \\
\text { sampling }\end{array}$ & 2272 & 5231 & 10480 \\
\hline
\end{tabular}

In Table III, the results for wind-hydro combined operation are presented. Not surprisingly, the combined wind-hydro approach gives results increasing the total profits over the wind-only case.

TABLE III

A Single DAY 1000 SCENARIO SiMULATION RESUlTS WIND-HYDRO TOTAL DAILY PROFITS

\begin{tabular}{c|c|c|c}
\hline $\begin{array}{c}\text { Scenario } \\
\text { gen. } \\
\text { method }\end{array}$ & $\begin{array}{c}\text { Min. } \\
\text { profit } \\
{[€]}\end{array}$ & $\begin{array}{c}\text { Avg. } \\
\text { profit } \\
{[€]}\end{array}$ & $\begin{array}{c}\text { Max. } \\
\text { profit } \\
{[€]}\end{array}$ \\
\hline $\begin{array}{c}\text { Hourly } \\
\text { indep. } \\
\text { sampling }\end{array}$ & 5163 & 6935 & 8866 \\
\hline $\begin{array}{c}\text { Time- } \\
\text { dep. } \\
\text { sampling }\end{array}$ & 2818 & 5867 & 11131 \\
\hline
\end{tabular}

Figures 7 and 8 give a graphical comparison of the results that can be related to the probability density function of $V A L$.

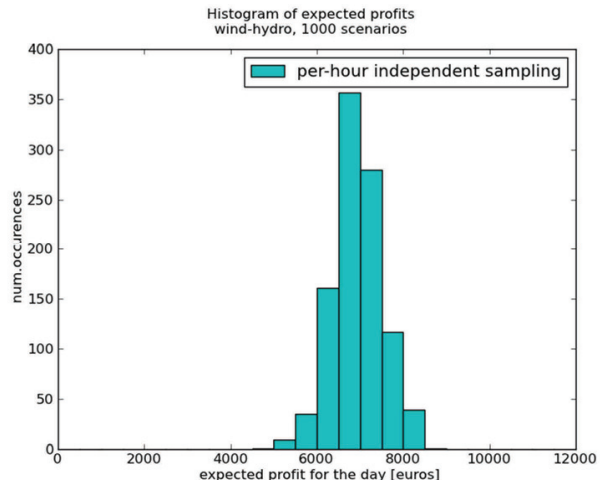

Fig. 7. A histogram illustrating the distribution of profit from optimizing 1000 wind power scenarios sampled using per hour independent scenario sampling

The non-linear transformation represented by the optimization model converting the forecast vector Pv to the random variable of profits VAL leads to extremely different shapes of the distribution of the latter.

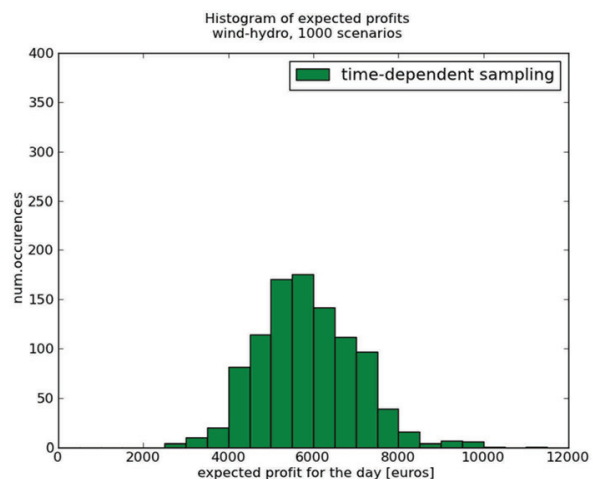

Fig. 8. A histogram illustrating the distribution of profit from optimizing 1000 wind power scenarios, using time-dependent scenario sampling

The two models are neither similar nor equivalent in consequences, so it is important to assess which is more faithful when wind power is taken into account. The distinguishing characteristic of each model is a set of generated scenarios taken as a representative of wind power uncertainty. To indicate the "representativeness" of the scenario sets, the profit maximization results are compared to the results of profit optimization based on perfect forecast.

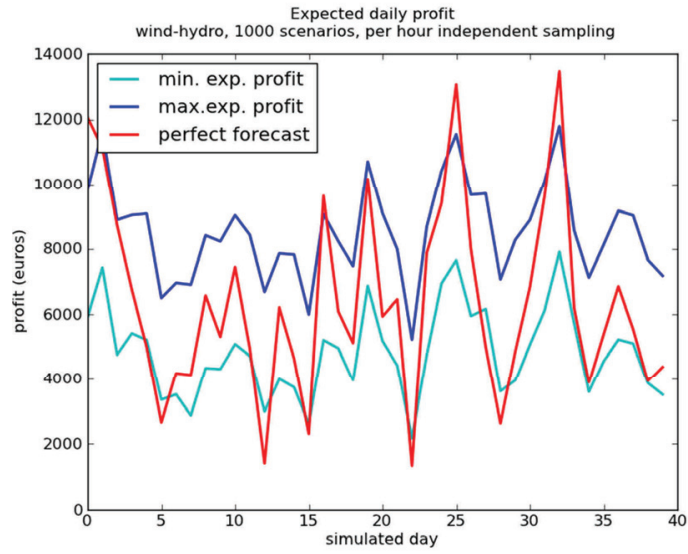

Fig. 9. Time series of the profit maximization results for 1000 wind power scenarios, using hourly independent scenario sampling (first 40 days)

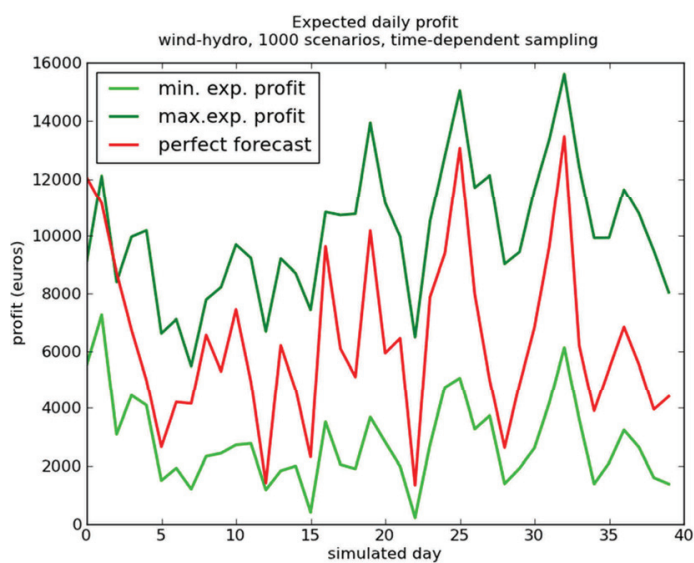

Fig. 10. Time series of the profit maximization results for 1000 wind power scenarios, sampled using time-dependent scenario sampling (first 40 days) 
The simulation is performed for 180 days (July-December 2006). For each of these days, 1000 scenarios are sampled using both the models and the results compared to the result obtained with the profit based on perfect forecast. Figures 9 and 10 illustrate the results (for 40 days). In each of these figures, the uncertainty band defined by the minimum and maximum of daily profits is depicted, as well as time series of "utopia" profits based on perfect forecasts. Examining both figures, it is obvious that the hourly independent sampling model fails to encompass the ideal profit obtained by a perfect prediction, i.e. this series has many points outside the range retrieved by the simulation. This is not the case when timedependent scenario generation is used. Table IV compares the number of hits and misses for both scenario generation techniques, for the 180 simulated days. A "hit" is considered when the expected daily profit based on perfect foresight falls within the extents of the profit band predicted by the scenario sampling method.

TABLE IV

CAPTURING THE DAILY PERFECT ForeCASt PROFIT WithiN THE RANGE OF WIND-HYDRO EXPECTED DAILY PROFITS

\begin{tabular}{c|c|c}
\hline $\begin{array}{c}\text { Scenario } \\
\text { gen. } \\
\text { method }\end{array}$ & Hits & Misses \\
\hline $\begin{array}{c}\text { Hourly } \\
\text { indep. } \\
\text { sampling }\end{array}$ & 131 & 49 \\
\hline $\begin{array}{c}\text { Time- } \\
\text { dep. } \\
\text { sampling }\end{array}$ & 174 & 6 \\
\hline
\end{tabular}

\section{CONCLUSION}

The conclusion is unequivocal: a method based on independent Gaussian assumption for hourly wind power forecasts is non-satisfactory. For the operational purposes when the modeled system includes storage, this paper reinforces the conviction: one should employ an uncertainty modeling method capable of embedding the temporal interdependence structure of uncertainty - neglecting this interdependence structure may lead to strong under- or overestimation of the uncertainty.

In this paper the focus was on a comparison of two modeling approaches for wind power forecast uncertainty in the daily optimization of a wind power plant combined with small-scale pumped hydro storage. The simplified approach of adding Gaussian noise to the point forecasts independent per each hour is compared to a method that transforms the shape of predictive distribution to multivariate Gaussian where the time interdependence structure is stored within an estimated covariance matrix.

A simplified setup of the simulation based on a realistic wind power forecast data confirms the hypothesis that a method that considers the temporal interdependencies performs favorably to the simple method that neglects these dependencies. This conclusion has implications on the process of determining the operational policies for a W-H power plant: in other words, correct estimation of the risk exposure cannot be based on an overly simplistic model of input uncertainty.
The adoption of a correct uncertainty estimation method is the key to a correct prediction of effects, namely economic, of operation planning decisions. This warning is especially relevant if one takes in account that uncertainty prediction models for wind power usually provide results where the uncertainty assessment, made at each hour, is void of temporal dependencies (they are marginal distributions at each hour). Departing from such a model to derive operation policies may lead to unfavorable economic results and even put the system at risk.

\section{ACKNOWLEDGMENTS}

Jean Sumaili acknowledges the Portuguese Fundação para a Ciência e a Tecnologia (FCT) for funding within Ciencia 2008 program. Hrvoje Keko acknowledges the same institution for PhD scholarship SFRH/BD/43087/2008.

\section{REFERENCES}

[1] C. Monteiro, R. Bessa, V. Miranda, A. Botterud, J. Wang, and G. Conzelmann, "Wind Power Forecasting: State-of-the-Art 2009." Argonne National Laboratory, 06-Nov-2009.

[2] Decreto-Lei.$^{\circ}$ 225/2007. DR 105 SÉRIE I, Portugal (Regulation on Remuneration of Renewables). 2007.

[3] R. J. Bessa, V. Miranda, A. Botterud, and J. Wang, "'Good' or 'bad' wind power forecasts: a relative concept," Wind Energy, 2011.

[4] E. D. Castronuovo and J. A. P. Lopes, "On the optimization of the daily operation of a wind-hydro power plant," Power Systems, IEEE Transactions on, vol. 19, no. 3, pp. 1599-1606, 2004.

[5] J. Juban, L. Fugon, and G. Kariniotakis, "Uncertainty estimation of wind power forecasts: Comparison of Probabilistic Modelling Approaches," 2008. [Online]. Available: http://hal-ensmp.archivesouvertes.fr/hal-00506297. [Accessed: 12-Oct-2010].

[6] J. B. Bremnes, "Probabilistic wind power forecasts using local quantile regression," Wind Energy, vol. 7, no. 1, pp. 47-54, 2004.

[7] P. Pinson and G. Kariniotakis, "On-line assessment of prediction risk for wind power production forecasts," Wind Energy, vol. 7, no. 2, pp. 119-132, 2004

[8] P. Pinson, H. Madsen, H. A. Nielsen, G. Papaefthymiou, and B. Klöckl, "From probabilistic forecasts to statistical scenarios of shortterm wind power production," Wind Energy, vol. 12, no. 1, pp. 51-62, 2009.

[9] B. Klöckl, G. Papaefthymiou, and P. Pinson, "Probabilistic tools for planning and operating power systems with distributed energy storage," e \& $i$ Elektrotechnik und Informationstechnik, vol. 125, no. 12 , pp. 460-465, 2008.

[10] R. J. Bessa, V. Miranda, and J. Gama, "Entropy and Correntropy Against Minimum Square Error in Offline and Online Three-Day Ahead Wind Power Forecasting," Power Systems, IEEE Transactions on, vol. 24, no. 4, pp. 1657-1666, 2009.

[11] G. Papaefthymiou and D. Kurowicka, "Using Copulas for Modeling Stochastic Dependence in Power System Uncertainty Analysis," Power Systems, IEEE Transactions on, vol. 24, no. 1, pp. 40-49, 2009.

[12] Enernex Corp., "Eastern Wind Integration and Transmission Study (EWITS)." Jan-2010.

[13] M. Brower, Development of eastern regional wind resource and wind plant output datasets. NREL - National Renewable Energy Laboratory, 2009. 